\title{
La circunferencia de la cintura en niños con sobrepeso y obesidad
}

\author{
JAIME PAJUELO ${ }^{1}$, ESTHER CANCHARI $^{2}$, JHON CARRERA $^{3}$, DIÓMEDES LEGUÍA ${ }^{2}$ \\ ${ }^{1}$ Instituto de Investigaciones Clínicas, UNMSM. ${ }^{2}$ Universidad Nacional Mayor de San Marcos. \\ ${ }^{3}$ Universidad Nacional de Trujillo.
}

\begin{abstract}
Resumen
Objetivo: Determinar e identificar a los niños que tienen otro riesgo adicional, como es el aumento de la grasa visceral. Material y Métodos: El estudio se llevó a cabo en Centros Educativos Estatales del distrito de Uripa (Apurímac), Trujillo (La Libertad) y Lima. La población estudiada fue de 1234 niños entre 6 a 10 años de edad. El 48,6\% correspondió al género femenino. A todos los niños se les tomó las siguientes medidas: peso, talla y circunferencia de la cintura (CC). Con las primeras se calculó el índice de masa corporal (IMC). Los valores del IMC fueron analizados con la clasificación de Must y col, identificando el sobrepeso y la obesidad a todos aquellos cuyo IMC se encontrara entre el 85 y 95 y más de 95 percentil, respectivamente. Los valores de la CC fueron analizados con las tablas de Freedman y McCarthy. Resultados: La prevalencia de sobrepeso y obesidad fue 16,5 y 13,9\%, respectivamente. El sobrepeso y obesidad, en su conjunto, disminuye conforme avanza la edad. El porcentaje de niños que tuvieron una CC por encima del 90 percentil se incrementó de acuerdo a la edad en que los niños presentaban sobrepeso y obesidad. El promedio de CC de todos los niños fue ligeramente mayor que la de los niños de EU de A y el RU.Conclusiones: Más de la mitad de los niños que tiene sobrepeso y obesidad presenta otro riesgo adicional, al tener la CC por encima de lo normal. Esta sumatoria de riesgos implicaría futuros problemas cardiovasculares, si no se toma las medidas correctivas inmediatamente.
\end{abstract}

Palabras clave: Obesidad;niño; índice de masa corporal; peso corporal; aumento de peso.

\section{Waist circumference in overweight and obese children Abstract}

Objective: To determine children with additional risk consisting in visceral fat increase. Material and Methods: The study was carried out at Uripa district (Apurímac), Trujillo (La Libertad), and Lima public schools. The population studied consisted in 1234 children 6 through 10 year-old; 48,6\% were female. Weight, height and waist circumference (WC) were done in all children and body mass index (BMI) was calculated with the first ones. BMI values were analyzed with Must et al classification, identifying overweight and obesity when BMI was 85 through 95 and above 95 percentiles, respectively. Waist circumference values were analyzed using Freedman and McCarthy tables. Results: Prevalence of overweight and obesity were 16,5 and 13,9\%, respectively. Overall, overweight and obesity decreased with increasing age. The percentage of children with WC above 90 percentile increases with age in overweight and obese children. Average WC was slightly higher than in both UK and USA children. Conclusions: Over fifty percent of overweight and obese children appeared to have an additional risk if their WC was above normal. Risks could imply future cardiovascular problems if corrective actions are not taken immediately.

Key words: Obesity; child; body mass index; body weight; weight gain.

\section{INTRODUCCIÓN}

El sobrepeso y la obesidad representan un problema que preocupa a los países desarrollados. En países como el nuestro, no se le ha dado la importancia que merece, por cuanto se tiene la percepción equivocada que esta patología está relacionada a los países desarrollados, lo que no es necesariamente así. 
El sobrepeso y la obesidad en mujeres adultas se ha incrementado de $36,7 \%$ en el año $1975\left(^{1}\right)$ a $46,7 \%$ para el año $2000\left(^{2}\right)$. Este hecho se encuentra enmarcado dentro de la tendencia epidemiológica que señala que estas enfermedades tienden a incrementarse. Chinn lo reporta así para el Reino Unido $\left({ }^{3}\right)$ y Strauss en EU de A $\left({ }^{4}\right)$.

Lo mencionado anteriormente no es patrimonio de los adultos. Lo más preocupante es que ya se está viendo en niños y que en este grupo la tendencia epidemiológica es también a incrementarse, conforme lo observado por Troiano ( $\left.{ }^{5}\right)$, Hanley ( $\left.{ }^{6}\right)$ y Reilly ( ). En el Perú, existen trabajos que reportan la presencia de estas enfermedades en niños de clases socioeconómicas altas ${ }^{8}$ ) e incluso en niños que asisten a Centros Educativos Estatales, tanto de las áreas urbanas $\left({ }^{8}\right)$ como en las rurales $\left({ }^{9}\right)$.

Independientemente de esto, existe en estos grupos otro riesgo que se relaciona a la presencia de un trastorno en los lípidos, lo que implicaría un mayor peligro para estos niños $\left({ }^{10}\right)$.

Si bien el IMC, cuando está incrementado, no determina si es a predominio de la masa magra y/o grasa, es menester usar los indicadores antropométricos que identifican los depósitos grasos y, entre éstos, la circunferencia de la cintura es el elegido, por cuanto señala a la grasa visceral, que es la que se relaciona directamente con los problemas cardiovasculares.

El objetivo del presente estudio es determinar e identificar a los niños que tienen otro riesgo adicional, como es el aumento de la grasa visceral.

\section{MATERIAL Y MÉTODOS}

El estudio se realizó en Centros Educativos Estatales. Se tomó la información de tres trabajos de investigación realizados en el distrito de Uripa (provincia de Chincheros, departamento de Apurímac) $\left({ }^{11}\right)$, en Trujillo (departamento de La Libertad) $\left({ }^{12}\right)$ y en Lima $\left({ }^{13}\right)$. La información fue recolectada en el segundo semestre de 2002 y en el primer semestre de 2003. En todos estos trabajos, se siguió la misma metodología para la toma de la información.

La población estudiada se agrupó en niños de 6 a 10 años. El total de niños alcanzó al número de 1234 , siendo $48,6 \%$ del género femenino.

Como indicadores antropométricos, a los niños se les tomó las siguientes medidas: peso, talla y circunferencia de la cintura. Con el peso y la talla se calculó el índice de masa corporal (IMC), que se expresa en kilogramos por metro cuadrado. La metodología aplicada para el peso, la talla y la circunferencia de la cintura es la recomendada por Lohman $\left({ }^{14}\right)$. Para la circunferencia de la cintura, se tomó en cuenta el borde inferior de la última costilla y el borde superior de la cresta iliaca; en la mitad de esta distancia se marcó, en ambos costados, para colocar la cinta métrica, esperando que el niño esté finalizando una espiración no forzada.

Para el uso de los valores encontrados de IMC con fines diagnósticos, se utilizó la tabla percentilar de Must y col $\left({ }^{15}\right)$, tomando en cuenta que, por debajo del 5, entre el 5 y 15,15 a 85,85 a 95 y más del 95 percentil, definen el déficit, peso bajo, normalidad, sobrepeso y obesidad, respectivamente.

En lo que concierne a los valores de circunferencia de la cintura, se los ha comparado con los valores encontrados por Freedman en el Bogalusa Heart Study ${ }^{(16)}$ y McCarthy $\left({ }^{17}\right)$.

Para el análisis estadístico, se ha utilizado el paquete estadístico SPSS 10.

\section{RESULTADOS}




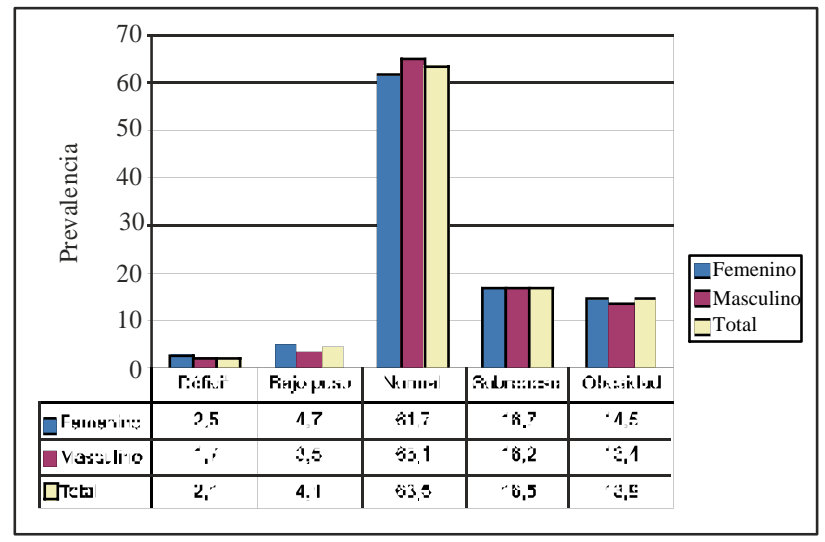

Tabla 1. Situación nutricional de niños de 6 a 10 años de Centros Educativos Estatales.

El Figura 1 permite apreciar la situación nutricional de los niños. Destaca nítidamente que la mayor presencia de patología se encuentra en los niños con sobrepeso $(16,5 \%)$ y obesidad $(13,9 \%)$, en relación a los de peso bajo $(4,1 \%)$ y déficit $(2,1 \%)$. En lo que respecta al género, prácticamente no hay diferencias significativas.

En el Figura 2, se puede observar la prevalencia de sobrepeso y obesidad por edades y género. En el grupo de 6, 7 y 8 años se presenta mayor prevalencia en el género femenino; lo contrario sucede en el género masculino. También se observa que, a mayor edad disminuye la

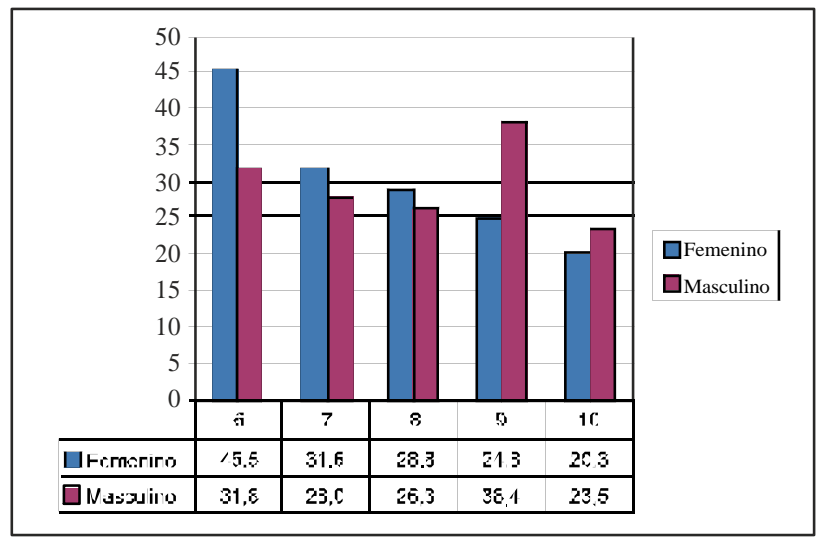

Tabla 2. Prevalencia de sobrepeso y obesidad por edad y género. prevalencia.

Para la elaboración del Figura 3, se ha utilizado sólo a los niños que se encuentran con sobrepeso y obesidad y cuya circunferencia de la cintura tuviera valores que se encontraran por encima del valor que corresponde al 90 percentil de lo hallado por McCarthy $\left({ }^{17}\right)$. No todos los niños de 6 años presentan el riesgo de tener incrementada la grasa visceral; pero sí, todos los de 10 años tienen -aparte del riesgo del sobrepeso y obesidad-, el riesgo del incremento de la grasa visceral.

La Tabla 1 muestra el promedio y desviación estándar de la circunferencia de la cintura por género. Asimismo, se puede observar lo mencionado en niños del Reino Unido (RU) ${ }^{\left({ }^{7}\right.}$ ) y de los Estados Unidos (EU de A) $\left({ }^{16}\right)$. Como se puede apreciar, los valores encontrados en el estudio son mayores que los reportados en los países mencionados. Por otro lado, la CC se incrementa conforme lo hace la edad.

\section{DISCUSIÓN}

En 1956, Vague $\left({ }^{18}\right)$ observó que los pacientes que mostraban un patrón de obesidad de la parte central o superior del cuerpo tenían una predisposición a la diabetes mellitus tipo 2 , la aterosclerosis y

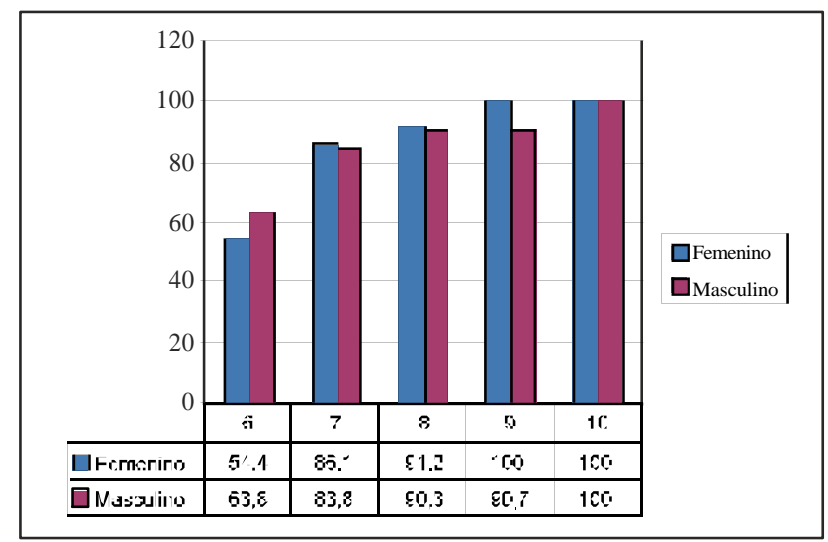

Tabla 3. Porcentaje de niños con sobrepeso y obesidad que presentan una circunferencia de la cintura por encima del $90 \mathrm{p}$. 
Jaime Pajuelo, Esther Canchari, Jhon Carrera, Diómedes Leguía

Tabla 1. Promedio y DE de la circunferencia de la cintura por género y edad

\begin{tabular}{|c|c|c|c|c|c|c|c|c|c|}
\hline \multirow[b]{2}{*}{ Edad } & \multicolumn{3}{|c|}{ FEMENINO } & \multirow[b]{2}{*}{ US A } & \multirow[b]{2}{*}{ Edad } & \multirow[b]{2}{*}{ No. } & \multicolumn{3}{|c|}{ MASCULINO } \\
\hline & No. & Media (DE) & $\mathrm{UK}$ & & & & Media /DE) & U K & US A \\
\hline 6 & 143 & $55.1(5.9)$ & $52.2(3.8)$ & 53 & 6 & 148 & $55.8(6.3)$ & $52.8(3.7)$ & 54 \\
\hline 7 & 114 & $58.8(7.1)$ & $54.4(4.7)$ & 54 & 7 & 132 & $59.3(5.6)$ & $54.6(4.0)$ & 55 \\
\hline 8 & 118 & $61.0(6.5)$ & $55.3(4.6)$ & 58 & 8 & 118 & $62.2(7.3)$ & $55.7(4.8)$ & 59 \\
\hline 9 & 109 & $62.6(6.7)$ & $56.8(4.9)$ & 60 & 9 & 112 & $65.6(8.3)$ & $58.1(5.6)$ & 62 \\
\hline 10 & 113 & $63.7(8.8)$ & $58(5.5)$ & 63 & 10 & 123 & $67(9)$ & $59.6(5.7)$ & 64 \\
\hline
\end{tabular}

la gota. En estudios longitudinales de población, realizados con varones y mujeres en Gothenburg, Suecia, que se llevaron a cabo entre 1967 y 1982, se encontró que la obesidad central predecía la aparición de diabetes mellitus, infarto de miocardio, angina de pecho, accidente cerebro vascular y muerte $\left({ }^{19}\right)$.

El exceso relativo de tejido adiposo en la región abdominal está asociado a concentraciones incrementadas de lípidos e insulina $\left({ }^{16}\right)$. Esta información puede ayudar a identificar a niños que posiblemente tienen efectos adversos a los lípidos e insulina.

Se ha reportado que la grasa visceral se encuentra significativamente relacionada al colesterol, triglicéridos y a la insulina basal, en el género femenino $\left({ }^{20}\right)$; por otro lado, la $\mathrm{CC}$ es bastante sensible y específica para identificar a niños con riesgo de desarrollar complicaciones metabólicas $\left({ }^{21}\right)$.

La circunferencia de la cintura es un indicador de grasa visceral. Su incremento no sólo refleja el incremento de la mencionada grasa, sino que también representa un aumento de la grasa subcutánea. En contraste, el IMC es la sumatoria de la masa grasa y masa magra, siendo imposible conocer la contribución de estos compartimentos cuando el IMC se encuentra por encima o por debajo de lo considerado normal $\left({ }^{22}\right)$. Es por esta razones que la $\mathrm{CC}$ es un mejor indicador que el IMC.

Existen varios trabajos que avalan que el mejor indicador antropométrico de riesgo cardiovascular es la circunferencia de la cintura $\left({ }^{23}\right)$. Daniels $\left({ }^{24}\right)$ reporta que la CC puede ser el indicador antropométrico más usado, en comparación con los resultados encontrados con absorciometría de energía dual de rayos X (DEXA).

En un trabajo en el Reino Unido, McCarthy $\left({ }^{25}\right)$ demostró el incremento de la prevalencia del sobrepeso y obesidad en niños en los últimos 20 años y hace especial referencia a que este aumento se ha hecho fundamentalmente en base a grasa visceral, lo que implica un riesgo mayor. Este hecho de por sí amerita reconocer que la CC debe ser una medida obligatoria como para incluirla en la rutina de la atención. En adultos, las poblaciones que tienen una mayor CC presentan porcentualmente mayor resistencia a la insulina y la presencia de síndrome metabólico $\left({ }^{26}\right)$. Incluso en niños severamente obesos, la CC ha sido considerada mejor predictor de la insulina en ayunas que el IMC $\left({ }^{27}\right)$.

Llama la atención que los niños del estudio, que de alguna manera representan a las clases pobres del país, tengan mejores promedios de cintura que en países desarrollados, como el del Reino Unido y EU de A. Esto quizás podría ser explicado en función del estado nutricional de los niños, ya que para estos fines se ha utilizado al total de niños. Sin embargo, cuando nos referimos exclusivamente a los niños obesos ( $>$ de 95p), se ha reportado promedios diferentes entre niños de la misma edad y del mismo nivel social $\left({ }^{28}\right)$. 
Lo preocupante de los niños que presentan sobrepeso y obesidad es que el riesgo, en función de la grasa visceral, se incrementa conforme lo hace la edad. Así se aprecia que $100 \%$ de los niños de 10 años de edad con sobrepeso y obesidad tienen exceso de grasa visceral. Lo contrario sucede con los de 6 años. Lo interesante es que, si bien la prevalencia del sobrepeso y la obesidad va disminuyendo, la grasa visceral va en sentido contrario. Esta tendencia quizá se interprete como que la presencia del sobrepeso y la obesidad en los adolescentes va a ser menor, pero que todos ellos tienen una grasa visceral en exceso. Sin embargo este hecho amerita profundizar los trabajos para encontrar una explicación.

Los estudios realizados en el Perú muestran que la mayor prevalencia se encuentra en el grupo de niños de 6 a 9 años $(18,2 \%)(29)$ que en el grupo de 10 a 18 años $(7,9 \%)$ (30). Si se pudiese inferir lo encontrado en el presente estudio, se podría afirmar que en mucho más riesgo se encontrarían estos últimos. Sin embargo, aquellos que aparentemente presentan menos riesgo (6 a 9 años), lo contraerán con el tiempo, sí no se toma medidas correctivas.

En conclusión, la $\mathrm{CC}$ es un indicador antropométrico de gran utilidad para precisar el riesgo asociado a problemas cardiovasculares y trastornos metabólicos. Es por esta razón que, en todo estudio nutricional donde se emplee la antropometría para el diagnóstico del sobrepeso y obesidad, no debe faltar la circunferencia de la cintura.

\section{REFERENCIAS BIBLIOGRÁFICAS}

1. Pajuelo J. Estado nutricional del adulto en el Perú. Acta Médica Peruana. 1992;16:22-32.

2. Instituto Nacional de Estadística e Informática (INEI). Encuesta Demográfica y de Salud Familiar (ENDES 2000) Lima: INEI; 2001.

3. Chinn S, Rona R. Prevalence and trends in overweight and obesity in three cross sectional studies of British children.
BMJ. 2001;322:24-6.

4. Strauss R, Pollack H. Epidemic Increase in childhood overweight, 1986-1998. JAMA. 2002;286:2845-8.

5. Troiano R, Flegal K, Kuczmarski R, Campbell S, Johnson C. Overweight prevalence and trends for children and adolescents. Arch Paediatr Adolesc Med. 1995;149:108591.

6. Hanley A, Harris S, Gittelsohn J, Wolver T, Saksvig B, Zinman B. Overweight among children and adolescents in a native Canadian community: prevalence and associated factors. Am J Clin Nutr. 2000;71:693-700.

7. Reilly J, Dorosty A, Emmett P. Prevalence of overweight and obesity in British children: cohort study. Br Med J. 1999;319:1039.

8. Pajuelo J, Morales H, Novak A. El sobrepeso y la obesidad en niños de áreas úrbanas del Perú. Diagnóstico. 2001;40:2029.

9. Pajuelo J, Villanueva ME, Chavez J. El sobrepeso y la obesidad en niños de áreas rurales del Perú. An Fac Med. 2000;61:2016.

10. Pajuelo J, Rocca J, Gamarra M. Obesidad Infantil: características antropométricas y bioquímicas. An Fac Med. 2003;64(1):21-6.

11. Leguia D, Pajuelo J. Situación nutricional de niños y adultos en el distrito de Uripa, provincia de Chincheros, departamento de Apurimac. I Congreso Nacional de Medicina, Cirugía, Pediatría y Ginecología. Lima: Cuerpo Médico, Hospital Nacional Dos de Mayo; 2003.

12. Carrera J, Pajuelo J. Situación nutricional de niños de un nivel socio económico de Trujillo. I Congreso Internacional y VII Congreso Peruano de Nutrición. Lima: Sociedad Peruana de Nutrición; 2004.

13. Canchari E, Pajuelo J. Situación nutricional de niños y adolescentes de un nivel socioeconómico bajo de Lima Metropolitana. I Congreso Nacional de Medicina, Cirugía, Pediatría y Ginecología. Lima: Cuerpo Médico. Hospital Nacional Dos de Mayo; 2003.

14. Lohman T, Roche A. Anthropometric Standardization Reference Manual. Illinois, Champaign: Human Kinects Books; 1990.

15. Must A, Dallal G, Dietz W. Reference data for obesity: $85^{\text {th }}$ and $95^{\text {th }}$ percentiles of body mass index (wt/ht 2 )-a correction. Am J Clin Nutr. 1991;54:773.

16. Freedman D, Serdula M, Srinivasan S, Berenson G. Relation of circumferences and skinfold thicknesses to lipid and insulin concentrations in children and adolescent: the Bogalusa Herat Study. Am J Clin Nutr. 1999;69:308-17.

17. McCarthy H, Jarret K, Crawley H. The development of waist circumference percentiles in British children aged 5.016.9 y. Eu J Clin Nut. 2001;55:902-7.

18. Vague J. Obesities. London: John Libbey Company Ltda; 1998.

19. Lebovitz HE. Resistencia a la insulina. SaoPaulo: Sciencie Press Ltda; 2003.

20. Caprio S, Hyman L, McCarthy S, Lange S, Bronson M, Tamborlane W. Fat distribution and cardiovascular risk factors in obese adolescent girls: importance of the intraabdominal fat depot. Am J Clin Nutr. 1996;64:12-7.

21. Taylor R, Jones E, Williams S, Goulding A. Evaluation of 
waist circunference, waist-to-hip ratio and the conicity index as screening tools for high trunk fat mass, as measured by dual energy X ray absorptiometry in children aged 3-19 years. Am J Clin Nutr. 2000;72:490-5.

22. Maynard L, Wisemandle W, Roche A, Chumlea G, Guo S, Siervogel R. Childhood body composition in relation to body mass index. Pediatrics. 2001;107:344-50.

23. World Health Organization. Obesity: preventing and managing the global epidemic. Report of a WHO consultation on obesity. Geneva: World Health Organization; 1997.

24. Daniels S, Khuory P, Morrinson J. Utility of different measures of body fat distribution in children and adolescent. Am J Epidemiol. 2000;152:1179-84.

25. McCarthy D, Ellis S, Cole T. Central overweight and obesity in British youth aged 11-16 years: cross sectional surveys of waist circumference. BMJ. 2003;326:624-5.
26. Ascaso J, Romero P, Real J, Lorente R, Martínez-Valle R. Abdominal obesity, insulin resistance, and syndrome metabolic in a southern European population. Eur J Intern Med. 2003;14:101-6.

27. Bedogni G, Iughetti L, Ferrari M, Malavolti M, De Simone M, Fiori G, et al. Association of waist circumference and body mass index with fasting blood insulin in severely obese children: a cross sectional study. Diabetes Nutr Metab. 2002;15(3):160-4.

28. Pajuelo J. La obesidad infantil en el Perú. Lima: Facultad de Medicina-UNMSM; 2003.

29. Pajuelo J, Amemiya I. El uso del índice de Quetelet en el diagnóstico nutricional en niños. An Fac Med. 1996;57:103-8.

30. Pajuelo J. La obesidad en el Perú. Alimentación y Nutrición No. 1. Lima: Cuadernos de Nueva Perspectiva; 1997.

\section{Correspondencia:}

Dr. Jaime PajueloRamírez

Hospital Nacional Dos de Mayo. Cuerpo Médico

Parque de la Medicina Peruna $s / n$

Lima 1, Perú

Correo-e: saturse@speedy.com.pe 